\title{
El tratamiento de la malaria en el entorno COVID-19
}

\author{
Kim West ${ }^{1}$, Raquel González Juárez 2, *y Carolina Nanclares ${ }^{3}$ \\ Asesora de asuntos médicos de MSF; ORCID id: https://orcid.org/0000-0002-6206-9017 \\ 2 Responsable de Relaciones Externas de MSF; ORCID id: https://orcid.org/0000-0001-7407-6698 \\ 3 Referente de Enfermedades Tropicales y Enfermedades Tropicales Desatendidas de MSF; ORCID id: \\ https://orcid.org/0000-0002-9118-0208 \\ * Autor correspondencia: juaraquel.gonzalez@medicososinfronteras.org
}

DOI: https://doi.org/10.37536/RIECS.2021.6.S1.250

Resumen: Pasados once meses del inicio de la pandemia de COVID-19, se han confirmado más de 103 millones de casos en 188 países y el número de muertes se acerca a los 2,5 millones. Uno de los efectos colaterales de la COVID-19 es el impacto en la salud no relacionada con el propio coronavirus y el aumento de las necesidades de salud. Este artículo aborda los principales vectores que están afectando a la provisión de servicios de salud y se centra en la explicación de la adaptación de la prevención y el tratamiento de la malaria en un entorno COVID-19 por Médicos Sin Fronteras, mediante un ejemplo de la estrategia realizada en la ciudad de Batangafo (República Centroafricana). El mantenimiento de la gestión de los casos de malaria en condiciones seguras ha sido esencial en un contexto de COVID-19, y hemos adaptado los procedimientos para mantener nuestras actividades en este ámbito; en especial, adaptando la estrategia de quimioprevención estacional de la malaria (SMC) y reforzando la gestión comunitaria, adaptada a las medidas de PCI contra la COVID-19. Se ha puesto especial atención a las medidas de prevención y control de infecciones (PCI) para identificar y aislar a las personas con síntomas compatibles con COVID-19, mantenido las acciones dirigidas al control y tratamiento de la malaria.

Palabras Clave: Médicos Sin Fronteras, malaria, Batangafo, República Centroafricana, COVID-19, MDA, PCI, SMC

\begin{abstract}
After eleven months of the start of the COVID-19 pandemic, more than 103 million cases have been confirmed in 188 countries and the number of deaths is close to 2.5 million. One of the side effects of COVID-19 is the health impact unrelated to coronavirus itself and increased health needs. This article addresses the main vectors that are affecting the provision of health services and focuses on explaining the adaptation of malaria prevention and treatment in a COVID-19 environment by Doctors Without Borders, using an example of the strategy carried out in the city of Batangafo (Central African Republic). Maintaining the management of malaria cases in safe conditions has been essential in a coVID-19 context, and we have adapted procedures to maintain our activities in this area; in particular, adapting the strategy of seasonal chemoprevention of malaria (SMC) and strengthening community management, adapted to PCI measures against COVID-19. Particular attention has been paid to infection prevention and control (PCI) measures to identify and isolate people with COVID-19-compatible symptoms, with actions aimed at malaria control and treatment.
\end{abstract}

Key words: Doctors Without Borders, Malaria, Batangafo, Central African Republic, COVID-19, MDA, PCI, SMC 


\section{Introducción}

Pasados once meses del inicio de la pandemia de COVID-19, se han confirmado más de 103 millones de casos en 188 países y el número de muertes ha superado los 2,2 millones ${ }^{1}$.

Aunque el impacto directo de la COVID-19 ha sido más lento y menos grave de lo esperado en muchos países donde Médicos Sin Fronteras (MSF) trabaja, especialmente en África, la pandemia sigue causando estragos en las vidas de las personas y es probable que lo siga haciendo en el futuro.

Numerosos grupos académicos y especializados han investigado los efectos colaterales de la COVID-19 en la salud no relacionada con el propio coronavirus. Estos análisis se han centrado principalmente en el efecto en los programas verticales de salud y predicen hambrunas de "proporciones bíblicas" 2 y escenarios con un aumento esperado de la mortalidad infantil de hasta un $45 \%{ }^{3}$. Si bien los datos que emergen de estos pronósticos han sido una clara llamada de atención para que Gobiernos, organizaciones no gubernamentales y donantes sigan manteniendo los programas no dirigidos a la COVID, en la práctica muchos programas esenciales de salud, protección, agua, saneamiento, higiene y medios de subsistencia se han reducido drásticamente en muchos países. Sin embargo, es difícil evaluar la magnitud de esta alteración y su efecto real en la salud y el bienestar de las poblaciones.

El impacto de la COVID-19 en las poblaciones está generando un aumento de las necesidades de salud. Desde que comenzó la pandemia, cada semana aparece una nueva investigación que informa del agravamiento de las necesidades de salud a consecuencia de la pandemia; por ejemplo, ha aumentado el número de mortinatos y las tasas de depresión se han triplicado4.

En los países de rentas bajas, preocupan especialmente las enfermedades prevenibles mediante vacunas, la seguridad alimentaria y la malaria, en gran parte debido a su impacto desproporcionado en la morbimortalidad y porque, debido a la COVID-19, se han suspendido muchas actividades preventivas relacionadas con estas áreas.

El impacto en las intervenciones contra la malaria es complejo, porque, además de la suspensión de las actividades de control de vectores (como la fumigación en interiores o la distribución masiva de mosquiteras tratadas con insecticida), también se han reducido las consultas prenatales, y es precisamente en estas consultas donde a menudo se entregan a las madres las mosquiteras y se dispensa el tratamiento de quimio prevención del paludismo. Todo ello se suma a la reducción de los servicios comunitarios y de atención primaria, que son la principal fuente de diagnóstico y tratamiento de la malaria ${ }^{5}$. Un estudio reciente del Fondo Mundial apuntaba el riesgo de que se produzcan 382.000 muertes adicionales por malaria en 2020 en comparación con 2018 debido a las interrupciones de los servicios relacionados con esta enfermedad ${ }^{6}$.

Otro estudio predice que, en los escenarios más pesimistas, en África podría casi duplicarse la mortalidad por malaria en 2020, con incrementos potencialmente mayores en los próximos años 7 .

Sin embargo, al estudiar casos concretos de país, hay informes contradictorios sobre el impacto real en los programas contra la malaria. Los datos de Nigeria, el país con más población amenazada por el paludismo (más de 200 millones de personas), sugieren que hasta ahora solo se han

\footnotetext{
1 A fecha de 1 de febrero de 2021

2 https://www.wfp.org/news/covid-19-will-double-number-peoplefacing-food-crises-unless-swift-action-taken

${ }^{3}$ https://papers.ssrn.com/sol3/papers.cfm?abstract_id=3576549

${ }^{4}$ https://www.nature.com/articles/d41586-020-02618-5. https://jamanetwork.com/journals/jamanetworkopen/ fullarticle/2770146

5 https://www.sciencemag.org/news/2020/08/scientists-worriedpandemic-would-cause-malaria-deaths-soar-so -far-it-hasnt-happened

6 https://www.theglobalfund.org/en/news/2020-06-24-global-fundcovid-19-report-deaths-from-hiv-tb-and-mal aria-could-almost-doublein-12-months-unless-urgent-action-is-taken/

7 https://www.thelancet.com/journals/laninf/article/PIIS1473-3099(20)30763-5/
} 
distribuido el 11,1\% de los 22,7 millones de mosquiteras previstas en 2020 y que la imposibilidad de continuar con las campañas dejaría sin mosquiteras a 34 millones de personas 8 .

Esto contradice una declaración reciente de la Alianza para Hacer Retroceder el Paludismo (Roll Back Malaria), según la cual siguen su curso más del $90 \%$ de las campañas contra la malaria programadas para este año en cuatro continentes ${ }^{9}$. Está claro que, si bien el impacto en los programas contra la malaria es específico a cada contexto, la COVID-19 podría estar añadiendo otra capa de desafíos en lugares donde combatir el paludismo ya es difícil debido a los conflictos o a la falta de acceso. Es algo a lo que MSF y otros actores de la salud deberían prestar mucha atención.

Las intervenciones contra la malaria también se están viendo negativamente afectadas por otros vectores que se están dando durante la COVID-19 y que no hacen sino dificultar un tratamiento y seguimiento adecuado de los casos de malaria:

\subsection{Acceso a instalaciones sanitarias}

A pesar del aumento de las necesidades, los servicios de salud también se han vuelto más escasos. Una encuesta reciente de la OMS reveló que el 90\% de los países han experimentado interrupciones de los servicios esenciales de salud relacionadas con la COVID-19, siendo más graves en los países de rentas bajas y medias ${ }^{10}$.

Algunos servicios se han visto más afectados que otros, pero la mayoría de los interrumpidos eran las vacunaciones rutinarias (tanto las externas $-70 \%$ - como en las estructuras de salud $-61 \%-$ ), el diagnóstico y tratamiento de enfermedades no transmisibles (69\%), la planificación familiar y los servicios anticonceptivos (68\%), la salud mental (61\%) y la atención prenatal (56\%).

Esta es una tendencia que MSF también ha visto en muchas de las zonas donde interviene. Por ejemplo, en Sudán, dos tercios de los centros de atención primaria están cerrados y, en el estado de Jartum, cerca del 70\% de las estructuras de salud llevan cerradas desde mayo ${ }^{11}$.

Mantener los proyectos de salud no relacionados con la COVID-19 ha sido la prioridad de MSF desde que comenzó la pandemia, pero nos hemos enfrentado a las mismas decisiones difíciles que los Ministerios de Salud y otras entidades del sector, y hemos tenido que reducir o interrumpir los servicios cuando, de hecho, necesitaban ampliarse más que nunca. En Sudán del Sur y la República Democrática del Congo, hemos tenido que cerrar proyectos antes de lo previsto; en Sudán y Burkina Faso, hemos tenido que reducir o interrumpir las consultas de atención primaria; y en casi todos los países hemos paralizado nuestros planes de ampliar cualquier servicio que no estuviera relacionado con la COVID-19.

Con tantos servicios interrumpidos, esto solo puede reducir drásticamente la atención médica disponible para la población. Esto, sumado a los confinamientos, no augura nada bueno. Las limitaciones de la atención médica comunitaria y primaria son particularmente preocupantes, dado que esta suele ser la primera interacción de una persona con el sistema de salud. En muchos lugares, los servicios se han cerrado sin dar información adecuada sobre las alternativas existentes, lo que significa que los pacientes llegan demasiado tarde o puede que ni lleguen a recibir atención.

Muchos factores explican la interrupción de los servicios de salud. Para MSF, y probablemente para muchos otros actores de la salud, las mayores limitaciones para continuar con nuestras operaciones se han relacionado con los recursos. La competencia mundial por los materiales básicos, en particular por los equipos de protección individual (EPI), supuso enormes dificultades para

\footnotetext{
8 https://gh.bmj.com/content/5/9/e003880

9 https://www.theguardian.com/global-development/2020/oct/02/malaria-campaigns-fight-off-covid-disruptio ns-to-deliver-programmes

10 https://reliefweb.int/sites/reliefweb.int/files/resources/WHO-2019-nCoV-EHS_continuity-survey-2020.1-eng. pdf

11 OCHA. 'Situation report'. Junio de 2020
} 
conseguir cantidades suficientes que garantizaran la seguridad y eficacia de los servicios médicos para pacientes y personal.

Las organizaciones han tenido que tomar decisiones muy difíciles sobre cómo priorizar programas y asignar recursos, desde el ámbito más global hasta el nivel del paciente individual ${ }^{12}$. Además, las restricciones de movimiento, los cierres de fronteras y las reducciones en el transporte comercial han impactado negativamente en las cadenas de suministro, provocando retrasos en la entrega y distribución y escasez de los productos y equipos esenciales necesarios para atender a los pacientes.

Los recursos humanos son, y siguen siendo, uno de los mayores desafíos en la prestación de atención médica, ya que las organizaciones médicas deben asegurar el equilibrio entre el deber de cuidar a su personal y el deber de cuidar a los pacientes.

MSF ha retirado de la primera línea de la atención a las personas más vulnerables a la COVID-19, lo que ha reducido el personal disponible para la atención directa del paciente. Este fenómeno se ha visto agravado por las restricciones de movimiento internacionales y nacionales, los retrasos en la emisión de visados y permisos, y los procedimientos de cuarentena y aislamiento.

\subsection{Calidad de la atención}

Es imposible proporcionar servicios de salud de manera segura y efectiva sin recursos suficientes, y una preocupación importante es de qué manera estas limitaciones están afectando a la calidad de la atención. Ha aumentado el intercambio y reparto de tareas entre perfiles sanitarios, con el fin de cubrir las necesidades, estrategia que normalmente se acompaña de supervisión y capacitación en persona ${ }^{13}$. Dadas las limitaciones de la distancia física, ha habido un cambio hacia la provisión de soluciones formativas a distancia o virtuales, pero la OMS considera que esto no reemplaza la capacitación in situ.

La prevención y el control de infecciones (PCI) es uno de los pilares clave de la calidad de la atención, y más que nunca en una pandemia de este tipo. Sin embargo, la PCI se ha visto afectada por la limitación de los recursos. Un ejemplo es que cerca del $14 \%$ de los casos de COVID-19 notificados a la OMS en todo el mundo se han producido en el personal de la salud, porcentaje que llega hasta el $35 \%$ en algunos países ${ }^{14}$. Nuestra experiencia en el terreno es que la cantidad de sanitarios con sospecha de contagio o contagio confirmado fluctúa de un mes a otro, pero el mayor porcentaje se observó en agosto, cuando el 26\% de los casos de COVID-19 en nuestros centros de salud eran de hecho sanitarios (no necesariamente personal de MSF).

\subsection{Implicación de la comunidad}

La COVID-19 también pone de manifiesto lo difícil que es para MSF comprender cómo manejan las personas su propia salud y bienestar cuando se reduce el acceso a las instalaciones médicas. Gran parte del trabajo de MSF se basa en que los pacientes y sus cuidadores acudan a las instalaciones. Pero si estas interacciones se reducen o se interrumpen, se complica saber cómo está afrontando la situación una determinada comunidad. La fragilidad de los contactos que tenemos con las personas con las que trabajamos se ha vuelto aún más pronunciada con grupos vulnerables, como mujeres y niños, desplazados internos y otras poblaciones en movimiento que huyen de la guerra o la violencia.

\footnotetext{
12 https:/www.nrc.no/globalassets/pdf/reports/make-or-break--theimplications-of-covid-19-for-crisis-financin g/nrc_make_or_break_implications_covid19_crisis_financing_ov.pdf

${ }^{13}$ https://www.cdc.gov/coronavirus/2019-ncov/global-covid-19/tasksharing.html

${ }^{14}$ https://uk.mobile.reuters.com/article/amp/idUSKBN2681TR?_twitter_impression=true
} 


\subsection{Desigualdad y pobreza}

Se espera que la crisis de la COVID-19 tenga el mayor impacto económico desde la Gran Depresión de la década de 1920; los pronósticos indican que la economía global se contraerá al menos un 4,9\% en 2020 y el Fondo Monetario Internacional (FMI) predice que podrían anularse los últimos diez años de progreso en la lucha contra la pobreza en los países de bajos ingresos ${ }^{15}$.

El consenso entre los principales economistas es que las personas más afectadas serán las más pobres y vulnerables y que la pandemia ahondará la desigualdad ya existente ${ }^{16}$.

Las estimaciones sugieren que entre 71 y 395 millones más de personas caerán en la pobreza extrema, la mayoría de ellas de países de África subsahariana donde no tienen protección social (el $80 \%$ de la población africana no está cubierta por una seguridad social $)^{17}$.

$\mathrm{Al}$ comienzo del brote, se prestó mucha atención al impacto de las medidas de contención en la economía de las familias, dado que muchas personas, en particular las que viven en zonas urbanas, trabajan en la economía sumergida. Sin embargo, el que algunos países relajen los confinamientos no significa que las oportunidades de empleo vayan a ser las mismas que antes de la COVID, ya que lamentablemente muchos negocios no volverán a abrir. Es probable que esta situación empeore cuanto más tiempo se vean afectadas las economías de los países por las restricciones. Los ingresos familiares continúan viéndose afectados porque las personas no pueden trabajar, debido a las cuarentenas y aislamientos, a la enfermedad, a las mayores responsabilidades de cuidado de los niños que no van a la escuela o porque se evita el trabajo por temor a infectarse. Muchas personas en los contextos donde trabaja MSF ya vivían al día antes de la COVID-19.

\section{El reto de abordar la malaria en un entorno COVID-19}

En los países donde trabajamos en zonas endémicas de África con una alta carga de paludismo, la prevención de la enfermedad es una de las inversiones más eficaces tanto para evitar la sobrecarga de los servicios sanitarios como para evitar la mortalidad por paludismo debida a una atención sanitaria disfuncional o colapsada. El mantenimiento de la gestión de los casos de malaria en condiciones seguras ha sido esencial en un contexto de COVID-19, y hemos adaptado los procedimientos para mantener nuestras actividades en este ámbito. MSF, a pesar de las dificultades anteriormente descritas, ha continuado trabajando en dos ejes esenciales: la prevención y la gestión de los casos.

En el ámbito de la prevención, hemos mantenido la distribución de mosquiteras por los canales habituales (consultas prenatales, vacunación, post-alta) cuando ha sido posible, pero hemos aplicado también nuevas estrategias adaptadas para asegurar la implementación de forma segura, respetando las normas de control y prevención de la infección, y garantizando el distanciamiento físico. Cancelar las distribuciones ha sido siempre la última opción, dado que pueden provocar más casos de malaria con la consiguiente aglomeración en los centros de salud, o muertes por falta de atención.

\footnotetext{
15 https:/www.nrc.no/globalassets/pdf/reports/make-or-break--theimplications-of-covid-19-for-crisis-financin g/nrc_make_or_break_implications_covid19_crisis_financing_ov.pdf

${ }^{16}$ https://blogs.imf.org/2020/05/11/how-pandemics-leave-the-pooreven-farther-behind/

$17 \mathrm{https} / /$ www.ids.ac.uk/opinions/social-impacts-of-covid-19-in-lowand-middle-income-countrie
} 
Otra de las estrategias que se ha priorizado implementar ha sido la quimio prevención estacional de la malaria (SMC por sus siglas en inglés). En marzo de 2012, la OMS recomendó SMC un tratamiento completo de sulfadoxina-pirimetamina + amodiaquina (SPAQ) en áreas con transmisión de malaria altamente estacional en la subregión del Sahel del África subsahariana, donde el $P$. falciparum es sensible a ambos medicamentos antipalúdicos. El tratamiento se dispensa una vez al mes durante 4 meses durante la temporada de transmisión para niños de entre 3 y 59 meses 18,19 .

Durante la pandemia de COVID-19, la estrategia de SMC se ha adaptado para evitar concentraciones de personas, realizando distribuciones puerta a puerta de SPAQ, con los EPI adecuados, el distanciamiento y el tratamiento directamente observado desde la primera dosis por el cuidador.

El tratamiento preventivo intermitente con sulfadoxina-pirimetamina (SP) durante el embarazo, recomendado a partir del primer trimestre en zonas de transmisión moderada o alta se debe reforzar evitando la aglomeración, por ejemplo, y aumentando los días en que se realizan las consultas prenatales. Si esto no es posible, se puede considerar la continuidad de la administración mensual de $\mathrm{SP}$ en el segundo y tercer trimestre a nivel comunitario cuando sea factible, por ejemplo, a través de redes comunitarias.

En cuanto a la gestión de los casos de malaria, ha sido vital mantener la gestión comunitaria (CCM), adaptada a las medidas de PCI contra la COVID-19. Los trabajadores sanitarios de la comunidad, incluso con una formación básica, desempeñan un papel crucial en el acceso a la atención médica. Se encargan de utilizar los test rápidos de diagnóstico (mRDT) para confirmar los casos sospechosos de paludismo, tratar los casos confirmados sin complicaciones y derivar a los pacientes con signos de alarma. Ha sido imprescindible incluir medidas de PCI, como el uso de EPI, y la adaptación y reducción del flujo de pacientes para reducir el riesgo de transmisión. Cuando estas circunstancias no han sido posibles, se ha recomendado el tratamiento combinado basado en la artemisimina a los pacientes con fiebre, respetando el distanciamiento seguro, práctica que acepta la OMS "en circunstancias excepcionales".

En el caso de pacientes con complicaciones, se realiza su derivación a centros sanitarios desde la comunidad, con el apoyo de MSF (bicicleta, motocicleta, etc.).

\section{Un ejemplo de caso: Batangafo, en República Centroafricana}

A pesar de todos los inconvenientes, en MSF hemos mantenido nuestro trabajo en el tratamiento de la malaria, especialmente en aquellos países o zonas donde es una de las principales causas de muerte entre los menores de 5 años, como en el caso de República Centroafricana, donde es la principal causa de muerte.

Como cada año, en septiembre llega la temporada de lluvias, y es cuando la malaria se vuelve más mortal que nunca en el país. La malaria en la RCA es holoendémica, con un pico estacional de junio a octubre. La tasa de positividad de las pruebas de diagnóstico rápido para la malaria en las consultas externas es de alrededor del $74 \%$ durante el pico y del $62 \%$ fuera del pico.

Teniendo en cuenta que la primera causa de morbilidad y mortalidad entre los niños en la RCA es la malaria, intentamos abordar el tema de la forma más completa posible, manteniendo la prevención y gestión de casos en niveles similares anteriores a la pandemia: a nivel curativo,

\footnotetext{
18 Seasonal Malaria Chemoprevention with sulfadoxine- pyrimethamine plus amodiaquine in children. A field guide. WHO. Fecha de publicación: 2013. ISBN: 9789241504737.

https://apps.who.int/iris/bitstream/handle/10665/85726/9789241504737_eng.pdf;jsessionid=576F6A85DC989869 084386F77D5D47C3? sequence=1

19 WHO Policy Recommendation: Seasonal Malaria Chemoprevention (SMC) for Plasmodium falciparum malaria control in highly seasonal transmission areas of the Sahel sub-region in Africa March 2012.
}

https://www.who.int/malaria/publications/atoz/smc_policy_recommendation_en_032012.pdf?ua=1 
aumentando el acceso a la identificación y el tratamiento precoz de los casos, tanto a nivel de estructuras de salud como a nivel comunitario. Las actividades comunitarias se intensificaron en 2020 para contrarrestar el descenso de visitas a los centros de salud. Asimismo, se aseguró un sistema de derivación de la periferia al hospital para los casos graves. En cuanto a la prevención, un aspecto fundamental es la distribución de mosquiteras tratadas con insecticida a los grupos más vulnerables (menores de 5 años y mujeres embarazadas).

Cada año, observamos un aumento de los casos de malaria en todos los proyectos de MSF en la República Centroafricana. En 2019, tratamos 578.072 casos de malaria en todo el país. Uno de nuestros proyectos se sitúa en la ciudad de Batangafo ${ }^{20}$, proporcionando apoyo en el hospital. Durante los periodos en que la transmisión de malaria es alta, ocho de cada 10 consultas pediátricas en el hospital son por malaria, incluyendo casos con complicaciones, como la anemia.

Se ha puesto especial atención a las medidas de prevención y control de infecciones (PCI) para identificar y aislar a las personas con síntomas compatibles con COVID-19, manteniendo las acciones dirigidas al control y tratamiento de la malaria.

Una de las estrategias implementadas en Batangafo ha sido la distribución masiva de medicamentos antimaláricos (MDA por sus siglas en inglés), con el objetivo de contribuir a la reducción de la mortalidad y morbilidad, principalmente relacionada con la malaria en la población $\mathrm{y}$, al mismo tiempo, reducir los casos de fiebre que pudieran presentarse en los centros de salud durante la pandemia de COVID-19, para evitar así la sobrecarga del sistema de salud

Esta estrategia se basó en la distribución mensual de un tratamiento completo basado en la artemisinina (artesunato + amodiquina en este caso) a toda la población durante el período de máxima transmisión de la malaria para tratar y prevenir la enfermedad. La población beneficiaria comprendió a todos los habitantes de la ciudad de Batangafo (8.065), más las personas asentadas en el campo de desplazados (22.542). En total, 30.607, de los cuales 12.800 eran niños y niñas menores de 14 años.

Los objetivos específicos de la intervención iban encaminados a:

- Reducir el número de casos y la mortalidad por paludismo en toda la población durante el periodo de alta transmisión (paludismo y COVID-19)

- Evitar la sobrecarga de los servicios relacionados con la malaria para garantizar una gestión de casos de calidad y una reducción de la asistencia a los centros (prevención de la exposición a la transmisión de COVID-19 a nivel de los centros sanitarios).

- Reducir el número de consultas por fiebre debidas a la malaria que podrían ser considerados como casos sospechosos de COVID.

Las actividades relacionadas con la administración masiva de medicamentos (MDA) se recomiendan en situaciones específicas como las epidemias y las emergencias complejas, en las que la capacidad del sistema se ve desbordada y no puede atender a las comunidades.

En cualquier caso, está claro que la prevención farmacológica no es la única forma de garantizar un buen resultado; hay que complementarla con medidas de distribución de mosquiteras (LLIN ${ }^{21}$ ) y de sensibilización sobre la importancia de su uso. Es importante señalar que esta estrategia se

\footnotetext{
${ }^{20}$ Ubicada en la prefectura de Ouham en el norte de RCA, la ciudad de Batangafo cuenta con 31.000 habitantes (22.000 de ellos desplazados internos) y ha sido un lugar de tensión política, étnica y religiosa durante más de una década. La situación de seguridad sigue siendo volátil y se espera que aumente la inestabilidad con las próximas elecciones, programadas para diciembre de 2020. Trabajamos en Batangafo desde 2006, apoyando al Ministerio de Salud en el hospital de Batangafo y en las áreas circundantes brindando atención de emergencia, cirugía, atención de maternidad, atención pediátrica, tratamiento para VIH/sida y tuberculosis, atención a supervivientes de violencia sexual y servicios de salud mental

${ }^{21}$ Long-lasting insecticidal nets
} 
propuso como temporal, teniendo en cuenta tanto el contexto de la pandemia de COVID-19, como sus posibles consecuencias en la saturación de los servicios de diagnóstico y tratamiento, así como para reducir las aglomeraciones en las instalaciones sanitarias.

Con el fin de llegar a un número máximo de personas y asegurarnos de que la población entendiera la importancia de esta iniciativa, la campaña se desarrolló en tres etapas.

a) Generación de conciencia sobre la campaña con la ayuda de los líderes comunitarios y transmisión de anuncios en la radio local. Antes, durante y después de la campaña, se realizaron actividades de promoción de la salud, sensibilización y movilización de la comunidad y de las poblaciones en las zonas de intervención. El principal objetivo fue aumentar el compromiso de la población con la toma de los medicamentos (adherencia). Se adaptaron los mensajes a diferentes grupos objetivo (líderes comunitarios, distribuidores comunitarios, supervisores, trabajadores sanitarios) y se realizaron mensajes adaptados para anticipar soluciones a los temores y preocupaciones de los beneficiarios, tales como la adaptación de la distribución del tratamiento en el entorno COVID-19; la importancia de la prevención del paludismo en un contexto de COVID-19; la aclaración de rumores y conceptos erróneos relacionados con los medicamentos (por ejemplo, que la MDA podía curar la COVID-19); o la explicación de los utensilios necesarios en los hogares antes de la campaña .

b) Visitas puerta a puerta para para distribuir el tratamiento preventivo. Al llevar el medicamento a las personas en sus propios hogares, evitaron el riesgo de que las multitudes se reunieran en los sitios de distribución y pudieran propagar la COVID-19. Nuestros equipos también adoptaron medidas de protección, como usar mascarillas y mantener una distancia segura entre las personas.

c) Seguimiento durante los días subsiguientes: visitas a cada hogar para para comprobar si las personas habían tomado el tratamiento e identificar cualquier efecto secundario.

La prevención y el tratamiento de la malaria es un eje fundamental del trabajo de MSF en los países endémicos, y a pesar de la COVID-19, el mantenimiento de estas actividades reducirá la mortalidad y la morbilidad por malaria, al tiempo que mejorará la posible confusión entre posibles casos de la COVID-19 y de malaria. 